\title{
A preliminary study of the hospital-admitted older patients in a Sri Lankan tertiary care hospital
}

\author{
$\mathbf{N}$ Weerasuriya ${ }^{1}$ and $\mathbf{S}$ Jayasinghe ${ }^{2}$
}

\begin{abstract}
Objective To provide reasons for admission, comorbidities, physical and psychosocial characteristics, and outcome of older patients admitted to hospital in Sri Lanka, and to assess adequacy of routine assessment.

Design Descriptive study.

Setting University Medical Unit, National Hospital of Sri Lanka during 10 weeks commencing August 2003.

Patients All $\geq 65$ years admitted to the unit, excluding transfers for specialised management and direct admissions by consultants or clinics; 75 men and 75 women.

Measurements Vision, hearing and speech, tests for tendency to fall, basic activities of daily living (BADL), mood and cognition, body mass index and waist circumference.

Results Seventy three per cent and $40 \%$ had cognitive dysfunction and depression., $20 \%$ required assistance in $\geq 5 / 9$ BADL, $23 \%$ had unprovoked falls within past year, and $57 \%$ had a tendency to fall, $34 \%$ had $\leq 6 / 60$ vision.

Conclusions Falls, depression, cognitive dysfunction and severe visual impairment were present in substantial proportions, unidentified by routine assessment of older patients.
\end{abstract}

\section{Introduction}

In Sri Lanka patients may seek treatment at tertiary centres without referral, and admission would be to a ward housing patients ranging in age from 13 to over 65 years. There is no special assessment and follow up of older patients for co-morbidities, impairments and disabilities. This may lead to inadequate care, recurrent admissions, and poor patient satisfaction. We studied the physical, psychosocial characteristics and outcome of 150 older patients admitted to our unit to assess the aforesaid parameters and the adequacy of current degree of clinical assessment.

\section{Method}

Consecutive admissions from the out-patient department who were $>65$ years old at the time of admission were studied. Exclusion criteria were admissions by consultants of the unit, referrals from the clinics and transfers for specialised management. Physical assessment included visual acuity (Snellen's chart), speech and hearing assessment, tendency to fall (timed up and go test) [1], and nutritional status (body mass index and waist circumference).

Status of the basic activities of daily living (BADL) was by Barthel's Index at the time of discharge. Cognitive function was screened by the three-item 5-minute recall test [2] and depression by the five-item geriatric depression scale $[3,4]$. Number of hospital admissions and unprovoked falls during the preceding year, marital status, financial and social support available, lodging, and social duties of the patient were recorded. The current case records were studied separately.

Statistical analysis was done by the Microsoft Excel Analysis Tool Pack with Chi test for testing significance of association and t-test for calculating significance with regard to measured variables.

\section{Results}

There were 75 women and 75 men. The mean age was 73 years (SD 5.9, 95\% CI 0.03). Mean duration of hospital stay was 4 days (SD 5.5). Five patients overstayed after discharge (range 1-43 days). Twelve patients died in the ward (8\%).

The common presentations were chest pain (17\%), breathlessness (14\%), fever (12.6\%), focal weakness $(11 \%)$, and dizziness (7\%). Diagnoses included ischaemic heart disease (IHD) (13\%), respiratory tract infection $(12 \%)$, stroke $(11 \%)$, urinary tract infection $(6 \%)$ and congestive cardiac failure (5\%). Five per cent were discharged pending further investigation. Coexisting illnesses were common, with $92 \%$ having one or more (average 2, maximum 6). More common co-morbidities were hypertension $(54 \%)$, IHD (30\%), osteoarthritis of the knees (29\%), diabetes mellitus (27\%) and bronchial asthma (12\%). Forty six per cent of the patients had one or more unplanned admissions to the hospital during the past year (mean 1.7, range $1-7$ ), and $48 \%$ of them had been within 6 weeks of the current admission.

Thirty four per cent had 6/60 or worse visual acuity bilaterally, $8.3 \%$ had impaired hearing, $7.6 \%$ had dysarthria and $2.2 \%$ had dysphasia. A $23.3 \%$ gave a history of unprovoked falls during the preceding year, and $57.6 \%$ could not or took longer than $30 \mathrm{~s}$ to perform the timed up and go test; $19.8 \%$ required assistance in 5 or more BADL, but $51.6 \%$ were independent with regard

${ }^{1}$ Senior Registrar, ${ }^{2}$ Associate Professor, University Medical Unit, National Hospital of Sri Lanka.

Correspondence: NW, e-mail: <namalw@yahoo.com> (Competing interests: none declared). Received 1 December 2003 and revised version accepted 19 August 2004. 
to all BADL. Seventy three per cent had cognitive dysfunction. Forty per cent had depression. Those living with their children or spouse were significantly less depressed than those who lived without them $(\mathrm{p}<0.05)$. Those having social duties were also less depressed than those without it $(\mathrm{p}<0.005)$.

Women were better nourished and had significantly lesser mortality than men, but there were no other significant differences (Table 1). Visual acuity, hearing, falls, BADL, weight (or height), cognitive function and mood had not been described in any patient on routine assessment.

Table 1. Comparison of sexes on physical and psychosocial parameters

\begin{tabular}{lrrr}
\hline & Men & Women & \multicolumn{1}{c}{$P$} \\
\hline Age (years) & 72 & 73 & 0.77 \\
Body Mass Index & 18 & 21.9 & 0.00003 \\
Waist circumference (cm) & 78 & 90 & 0.00002 \\
Cognitive dysfunction (\%) & 75 & 74 & 0.95 \\
Depression (\%) & 41 & 47 & 0.47 \\
Vision 6/60 or worse (\%) & 32 & 35 & 0.72 \\
Timed up and go test: $>30$ seconds (\%) & 55 & 61 & 0.47 \\
BADL: assistance required for $>5 / 9$ (\%) & 16 & 22 & 0.38 \\
Duration of stay in hospital (days) & 5 & 4 & 0.22 \\
Unplanned admissions in past year (\%) & 45 & 48 & 0.69 \\
Death during current admission (\%) & 13.3 & 2.6 & 0.02 \\
\hline BADL
\end{tabular}

BADL-basic activities of daily living, $\mathrm{P}$-using t-test for quantitative variables and Chi test for association between qualitative variables.

\section{Discussion}

Substantial numbers of older patients were identified with depression, possible cognitive dysfunction, an increased tendency to fall, poor vision and inability to care for themselves. These were not identified and addressed on routine management. This finding supports a structured and inclusive assessment schedule. Once introduced in the unit, an audit of its application and usefulness can be planned. Opportunities for wider inputs from psychogeriatrics and social services are being explored for improving the care of older patients admitted to the unit.

The reason for women being better nourished and having less mortality than men is difficult to explain, though it is possible that men delayed admission or treatment until they are more ill. High rates of recurrent admissions could be due to premature discharge, unaddressed comorbidities or the relapsing nature of certain illnesses.

This is a small study confined to one unit. Hence it is not possible to generalise its findings to the rest of the country, though it would also be unwise to assume that management of older patients is better elsewhere. The time may be right for an integrated geriatric service in Sri Lanka.

\section{References}

1. Podsiadlo D, Richardson S. The timed "Up and Go": A test of basic functional mobility for frail elderly persons. Journal of the American Geriatrics Society 1991; 39:142-8.

2. Siu AL. Screening for dementia and investigating its causes. Annals of Internal Medicine 1991; 115: 122-32.

3. Hoyl MT, Alessi CA, Harker JO, Josephson KR, Pietruszka FM, et al. Development and testing of a fiveitem version of the Geriatric Depression Scale. Journal of the American Geriatrics Society 1999; 47: 873-8.

4. Rinaldi P, Mecocci P, Benedetti C, Ercolani S, Bregnocchi $\mathrm{M}$, et al. Validation of the five-item geriatric depression scale in elderly subjects in three different settings. Journal of the American Geriatrics Society 2003; 51: 694-8.

\section{Choice of antibiotic}

If an antibiotic is indicated, a drug should be chosen that will limit the development of bacterial resistance. The choice of drug is influenced by the likely pathogens and local resistance patterns.

J Ferguson. Antibiotic prescribing: how can emergence of antibiotic resistance be delayed? Australian Prescriber 2004; 27: 39-42. 